(C) 2015 the authors. Access to this work was provided by the University of Maryland, Baltimore County (UMBC) ScholarWorks@UMBC digital repository on the Maryland Shared Open Access (MD-SOAR) platform.

Please provide feedback

Please support the ScholarWorks@UMBC repository by emailing scholarworks-group@umbc.edu and telling us what having access to this work means to you and why it's important to you. Thank you. 


\title{
Web Search Credibility Assessment for Individuals who are Blind
}

\author{
Ali Abdolrahmani, Ravi Kuber and William Easley \\ UMBC \\ aliab1@umbc.edu
}

\begin{abstract}
While screen reading technologies offer considerable promise to individuals who are blind by providing an accessible overview of web-based content, difficulties can be faced determining the credibility of sites and their respective contents. This can impact the user's behavior, particularly if sensitive information needs to be entered (e.g. into a web-based form). In this paper, we describe an exploratory study examining the criteria which blind screen reader users utilize to assess credibility. More specifically, we have focused on the common task of web searching and exploring search results. Findings from the study have suggested that mismatches between the title of the search results and their respective snippets, along with the richness and accessibility of the content when search results are selected, can lead to users determining whether sites are indeed credible.
\end{abstract}

\section{Categories and Subject Descriptors}

K.4.2 [Assistive technologies for persons with disabilities]

\section{General Terms}

Design, Human Factors

\section{Keywords}

Blind, Credibility, Screen reader, Trust, Web content, Web search

\section{INTRODUCTION}

The benefits of the Web are numerous. However, the presence and sometimes, prominence of incorrect and misleading content on the Web can have serious consequences [5]. Examples may include the consequences of divulging sensitive information via an online form, or trusting content about treating a medical condition after reading about it on a medical site. Visual cues, such as the 'look and feel' of content, are often used to determine credibility of a site. However, for individuals who are blind, the process is more complex. Screen readers are used to provide a representation of web-based content in auditory or tactile format. However, these tools are known to be restrictive in nature [1], presenting information sequentially, and inadequately handling graphical content. If the web page has been poorly coded or content is unclear, the browsing process will be negatively impacted.

In this paper, a study is described examining the ways in which individuals who are blind determine credibility of sites and their respective content when using a screen reader. More specifically,

Permission to make digital or hard copies of part or all of this work for personal or classroom use is granted without fee provided that copies are not made or distributed for profit or commercial advantage and that copies bear this notice and the full citation on the first page. Copyrights for thirdparty components of this work must be honored. For all other uses, contact the Owner/Author.

Copyright is held by the owner/author(s).

ASSETS '15, October 26-28, 2015, Lisbon, Portugal

ACM 978-1-4503-3400-6/15/10.

http://dx.doi.org/10.1145/2700648.2811349 we have focused on web search tasks, as these are commonly undertaken by both blind and sighted users. Findings can help to inform web designers interested in heightening levels of credibility among a more diverse audience.

\section{RELATED WORK}

According to Fogg [2], the concept of credibility is made up of two dimensions: trustworthiness and expertise. If a web site conveys both qualities, it would be considered credible. The researcher suggests that users may be influenced by their first impressions of inspecting a site, and whether it is professional in its design (termed: surface credibility). Trust may also be established over time, influenced by the site's ease of use and its ability to provide credible information consistently (termed: earned credibility). Schwarz and Morris [5] developed visualizations to augment search results and web pages in order to help users to more accurately judge the credibility of online content. Findings from the study highlighted the potential of data aggregation and simple interventions to help people make more informed decisions as they search for information online.

While research examining issues associated with web credibility has been valuable, there has been limited work examining the needs of individuals with diverse abilities (e.g. individuals who are blind), to determine whether their needs differ from a mainstream audience. Information seeking behavior is known to vary between blind and sighted users [4]. As it can be challenging to access visual cues through a screen reader, the process of establishing credibility can be complex. In the study described in this paper, we aim to explore the criteria that are used by individuals who are blind to make credibility judgments.

\section{EXPLORATORY STUDY}

Four congenitally blind participants (two females, aged $20-32$ ) were recruited for the study. All were experienced JAWS screen reader users, and used Google search engine as their preferred web search tool.

Two tasks were selected for the study: (1) conducting two searches based on a set of pre-defined search terms; (2) browsing five pairs of web pages exploring a set of pre-defined topics, including health and current affairs. During this process, participants were asked to 'think-aloud', walking through the process of searching the Web using the browser and screen reader of their choosing, and describing how levels of credibility could be established.

For the first task, participants were asked to identify answers to a particular question (e.g. "Who invented the screen reader?") and to undertake more open-ended searching (e.g. "What can you find out about the link between diabetes and blindness?). For the second task, each pair of pages referred to a similar topic (e.g. Ebola symptoms and treatment, energy saving tips for winter). However, each page differed in terms of the quality and volume of content and structural layout. Participants were instructed not to 
spend any longer than five minutes on each task. Comments made by participants, along with screen reader commands which were executed, were noted during the study. Data was then coded and analyzed.

\section{RESULTS AND DISCUSSION}

\subsection{Browsing Strategy}

Similarities between browsing styles were evident among participants. Techniques included 'skimming' through each of the search results, using the Control + Down Arrow shortcut to gain an overview. As search results were outputted, participants would listen out for keywords related to their search, in the hope that there would be information matching their query. This process required concentration. Three participants also used the ' $h$ command' to navigate through a list of headings, with the same aim.

\subsection{Credibility of Search Results}

- Verifying results are related to the intended search - After identifying appropriate headings using the ' $h$ command' described in 4.1, three participants checked the source URL associated with the search result, followed by the snippet. The snippet was used to verify that the search result heading was consistent with the search query keywords.

- $\quad$ Putting faith in the ranking of search results - Participants were generally trusting that search results are presented in an order of relevance to the term entered. $\mathrm{P} 4$ described browsing through the first sets of search results, as he felt the latter results would be less relevant to his original query and be time-consuming to read. If the results were irrelevant to his needs, he would access the 'Did You Mean' option, as this could help with the process if a spelling mistake had been made in the original search.

- Mismatches - When searching on a particular topic, participants identified mismatches between the search results and the respective text snippet. This resulted in time rereading both items using a screen reader, and then doublechecking the search box to ensure that they had searched on the correct terms. P3 pointed out that he would select the search result link to open the associated page, and check if the content on the page would match with the search result. Confusion would ensue if the back button was selected, as it would be difficult for him to find his previous location in the page of search results.

\subsection{Credibility of Web Sites}

- Domain Name - Familiarity with the organization whose site was being accessed, was the main criterion to determine the credibility of a site. If the information being sought (query) corresponded to the type of site where they would expect to read about this type of information, it would be easier for them to trust the content. For example, reading about Ebola symptoms on a well known medical web site (e.g. WebMD), or 2016 US election candidates in a respected venue (e.g. New York Times).

- Domain Type - All four participants found governmental and reputable organizations more credible compared to blogs, forums or pages that were opinion-based. As a result, domain types such as '.gov' would be considered to have greater levels of credibility, compared with '.biz'. However, participants highlighted that while they were aware that sighted users could glance at a URL to double-check the domain type being accessed, this would be a more timeconsuming process to perform using a screen reader.

- Accessibility of content -All four participants appeared to associate levels of credibility with site accessibility. For example, sites containing images without alt text or headings formatted without the appropriate tags, were thought to be hastily designed. If time could not be spent improving accessibility of the site, it was possible that time was not spent verifying the accuracy of textual content on the site. As a result, these were considered to have lower levels of credibility compared with other more accessible sites.

- Presence of extraneous content -All four participants assessed pages with less irrelevant content as of a more credible source. Sites with adverts and other pop-ups were considered less credible, and skipped where at all possible.

- Richness of content - Other factors which influenced participants included (1) the style of writing (i.e. whether casual or professional), (2) authors of the content (in the case of news stories), (3) whether or not the content was reviewed by an domain expert (e.g. P3 found that the article about the Ebola symptoms in WebMD was reviewed by a medical doctor), and (4) presence of other relevant article links. Experience of accessing web sites over time had enabled participants to make judgment calls on whether sites were credible. For example, all four expected a web site examining a scientific topic, to be written professionally, which in turn informed credibility.

\section{FUTURE WORK}

We aim to expand the study, recruiting a larger sample of both blind and sighted web users. The aim would be to determine the ways in which credibility perception and assessment varies between both groups. Similar to [3]. we aim to specifically examine the ways in which the structural layout of information can impact blind users, as the visual layout of content on an interface is known to impact sighted users [2,5]. Insights from such a study can provide guidance to developers interested in heightening web credibility among a wider range of users.

\section{ACKNOWLEDGMENTS}

The authors would like to thank Jenna Tart for her assistance with this research.

\section{REFERENCES}

[1] Chandrashekar, S., 2010. Is Hearing Believing? Perception of Online Information Credibility by Screen Reader Users who are Blind or Visually Impaired. Doctoral thesis, University of Toronto, Canada.

[2] Fogg, B.J. 2003. Persuasive Technology: Using Computers to Change What We Think and Do. Morgan Kaufmann.

[3] Leuthold, S., Bargas-Avila, J.A., and Opwis, K., 2008. Beyond web content accessibility guidelines: Design of enhanced text user interfaces for blind internet users. Int $J$ Hum Comput Stud 66, 4, 257-270.

[4] Sahib, N.G., Tombros, A. and Stockman, T., 2012. A comparative analysis of the information-seeking behavior of visually impaired and sighted searchers. J Assoc Inf Sci Technol, 63, 2 (Feb. 2012), 377-391.

[5] Schwarz, J. and Morris, M., 2011. Augmenting web pages and search results to support credibility assessment. In Proceedings of the SIGCHI Conference on Human Factors in Computing Systems, ACM, New York, NY, 1245-1254. 